\title{
Experimental Study on Dual-Wavelength Distributed Feedback Fiber Laser
}

\author{
Haifeng QI*, Zhiqiang SONG, Jian GUO, Chang WANG, \\ Jun CHANG, and Gangding PENG
}

Shandong Key Laboratory of Optical Fiber Sensing Technologies, Laser Institute of Shandong Academy of Sciences, Jinan, 250014, China

${ }^{*}$ Corresponding author: Haifeng QI $\quad$ E-mail: qihf@sdu.edu.cn

\begin{abstract}
Four distributed feedback fiber lasers with dual-wavelength operations were studied experimentally in detail. The laser characteristics of these distributed feedback fiber lasers (DFB-FLs) were investigated. The operation conditions for the dual-wavelength output are analyzed and presented. Through this study, the guidance of the design and fabrication of some dual-wavelength distributed feedback fiber lasers are provided.
\end{abstract}

Keywords: Distributed feedback fiber laser, dual wavelength, phase shift, optical fiber sensing

Citation: Haifeng QI, Zhiqiang SONG, Jian GUO, Chang WANG, Jun CHANG, and Gangding PENG, "Experimental Study on Dual-Wavelength Distributed Feedback Fiber Laser," Photonic Sensors, 2014, 4(3): 225-229.

\section{Introduction}

Dual-wavelength lasers are widely used in wavelength-division-multiplexing communications systems, sensing, lidar-radar systems, high-resolution interferometry, and microwave photonic generation. A lot of dual-wavelength fiber lasers with different structures have been reported [1-9], e.g. moiré distributed feedback (DFB) fiber laser [1], distributed Bragg reflector (DBR) fiber laser [2], chirped DFB fiber laser [3], cascaded DFB fiber laser [4], and sampling grating fiber laser [5]. The distributed feedback fiber laser (DFB-FL) attracts more attention for its compact dimension and ultra narrow linewidth since the first invention [10]. The dual-wavelength DFB-FL owning these above advantages has been achieved by introducing two phase shifts [6] or two equivalent phase shifts $[7,8]$ locally.
In this paper, the dual-wavelength DFB-FLs with different structure parameters are investigated experimentally. The DFB-FL samples presented in this paper are all dual-wavelength lasers, however, they show different characteristics. The relations of their laser characteristics to the structural and fabrication parameters are analyzed. The conclusions are presented for the design and fabrication of the dual-wavelength DFB-FL or single wavelength DFB-FL.

\section{Experiments}

Four phase-shifted gratings were written in four pieces of 6-cm-length photosensitive Erbium doped fiber (Nufern, the peak absorption at $1530 \mathrm{~nm}$ is $8 \mathrm{~dB} / \mathrm{m}$ ) by scanning $244 \mathrm{~nm}$ frequency-doubled harmonic Argon ion continuous wave laser across the phase mask and fiber (shown in Fig. 1). The scanning speed of the ultraviolet (UV) laser was

Received: 25 February 2014 / Revised version: 12 May 2014

C The Author(s) 2014. This article is published with open access at Springerlink.com

DOI: $10.1007 / \mathrm{s} 13320-014-0182-3$

Article type: Regular 
electrically controlled to obtain the desiring UV-induced index modulation amplitude. The phase mask was placed on a piezoelectric transducer (PZT) moving stage with the nm-grade resolution and accuracy. The phase shift was produced by an instantaneous movement of the phase mask to the fiber at the specific position, and the movement was $\Lambda / 4$ for a $\pi$ phase shift where $\Lambda$ is the period of the phase mask pitch. The polarization direction of the $244 \mathrm{~nm}$ UV laser was in the horizontal polarization, and it could be adjusted to the vertical polarization by turning the polarization controller. The structural parameters and the fabrication parameters are listed in Table 1.

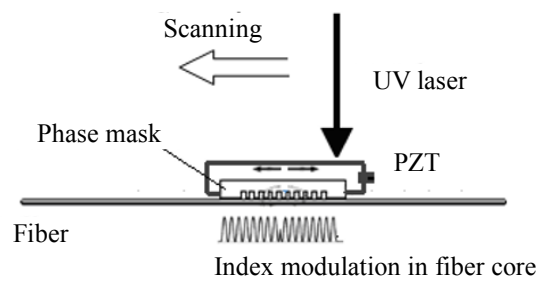

Fig. 1 Diagram of the experimental setup to fabricate the phase-shifted grating.

Table 1 Structural and fabrication parameters of the DFB-FLs.

\begin{tabular}{cccccc}
\hline Code & $\begin{array}{c}\text { Length } \\
(\mathrm{mm})\end{array}$ & $\begin{array}{c}\text { Phase } \\
\text { shift }\end{array}$ & PS position & $K^{*} L$ & UV polarization \\
\hline DFB-FL1 & 40 & Dual $\pi$ & $\begin{array}{c}\text { PP1 }=0.4 L \\
\mathrm{PP} 2=0.6 L\end{array}$ & $\sim 8$ & $\mathrm{~S}$ \\
DFB-FL2 & 40 & $\pi$ & Center & $\sim 13$ & $\mathrm{~S}$ \\
DFB-FL3 & 30 & $\pi$ & Center & $\sim 10$ & $\mathrm{~S}$ \\
DFB-FL4 & 40 & $\pi$ & Center & $\sim 8$ & $\mathrm{P}$ \\
\hline
\end{tabular}

Note: $K$ is the coupling coefficient of the grating.

There were two $\pi$ phase shifts in DFB-FL1, and the phase shifts were symmetrically around the center with the first at $0.4 \mathrm{~L}$ and the second at $0.6 \mathrm{~L}$, where $L$ is the phase-shifted grating length. For the other three DFB-FLs, the single discrete $\pi$ phase shift was at the center of the DFB-FL. The polarization of the UV laser to inscribe DFB-FL4 was parallel to the propagation axis of the fiber, i.e. P-polarization. The UV laser polarization for fabricating DFB-FL1, DFB-FL2, and DFB-FL3 was the vertical polarization, i.e. S-polarization.
The optical fiber grating was polarization dependent, and the UV induced birefringence was different for the P-polarization and S-polarization UV lasers. It has been studied and presented by several researchers $[11,12]$. The UV induced birefringence by the vertical polarization was much higher than that by the horizontal polarization (P-polarization) laser. Hence, there were different $\mathrm{UV}$ induced birefringences in DFB-FL1 to DFB-FL3 and DFB-FL4.

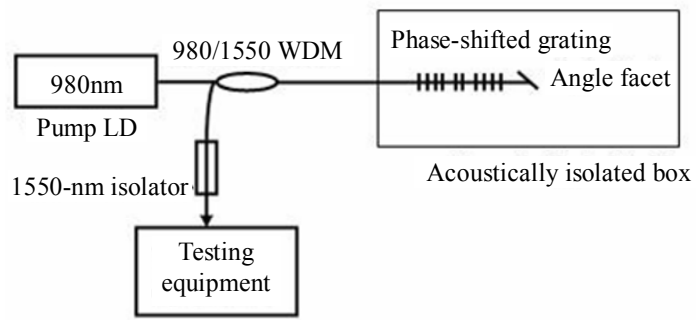

Fig. 2 Schematic diagram of the DFB-FL and the measurement.

\section{Results and discussion}

In our experiment, the four DFB-FLs all emitted dual-wavelength laser irradiation; however the specific characteristics were different from each other. The characteristics of the DFB-FLs, such as the laser spectrum, polarization state, and relative intensity noise, were tested systemically. The testing setup configuration is shown in Fig. 2. The laser spectra of the DFB-FLs measured with a high-resolution optical spectrum analyzer (Apex $2040 \mathrm{a}$, the wavelength resolution is $0.16 \mathrm{pm}$ ) under $100 \mathrm{~mW} 980 \mathrm{~nm}$ laser pump are shown in Fig. 3. The polarization characteristics of the DFB-FLs were measured with an optical component analyzer (Agilent N7788B). Their laser characteristics are summarized in Table 2 for comparison.

Table 2 Characteristics comparison for the 4 DFBf-FLs.

\begin{tabular}{cccc}
\hline Code & Wavelength gap & DOP & Comments \\
\hline DFB-FL1 & $20 \mathrm{pm}$ & 1 & Stable single polarization \\
DFB-FL2 & $9 \mathrm{pm}$ & 0.7 & Competing dual polarization \\
DFB-FL3 & $76 \mathrm{pm}$ & 1 & Stable single polarization \\
DFB-FL4 & $2 \mathrm{pm}$ & 0.06 & Stable dual polarization \\
\hline
\end{tabular}



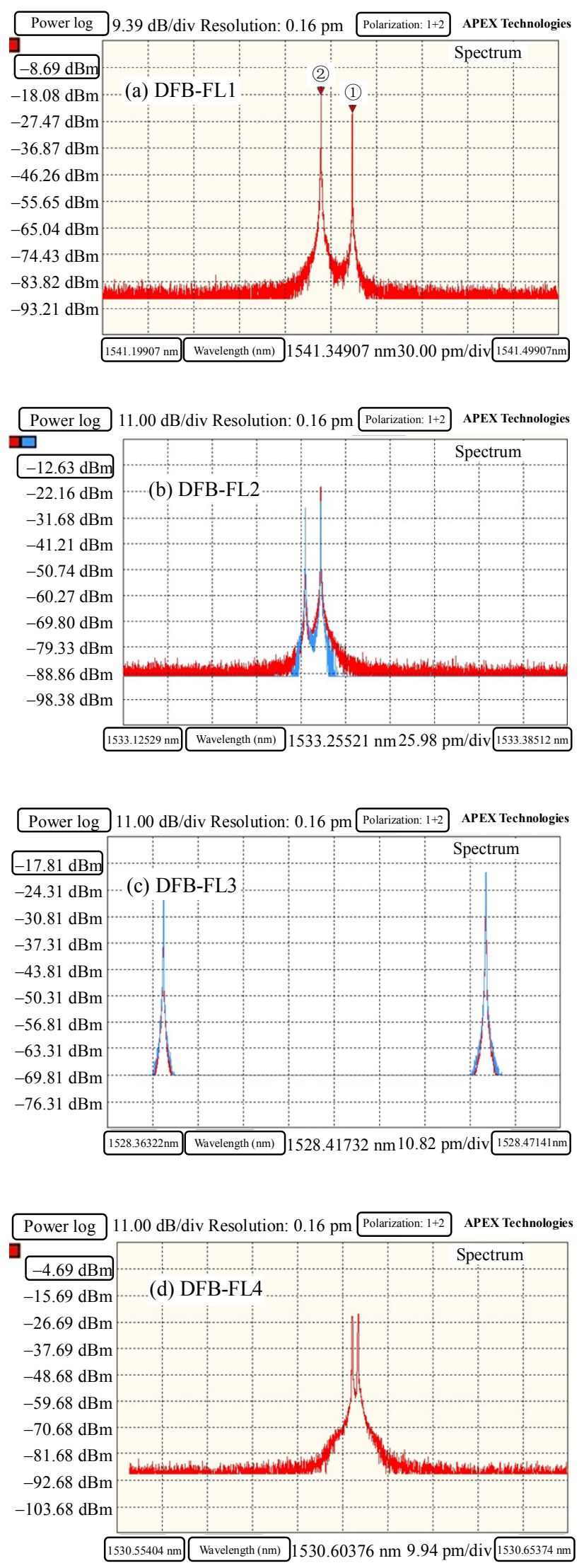

Fig. 3 Laser spectra of the four dual-wavelength DFB-FLs.
Based on the experimental results, we could see that the dual-wavelength lasers were all obtained in these DFB-FLs but had different performances. DFB-FL1 with 2 symmetrical $\pi$ phase shifts had the stable dual-wavelength laser irradiation with the single polarization. The two phase shifts in grating opened two symmetrical transmission slits in the Bragg reflection band of the grating without consideration with the polarization. The simulation can be found in [13]. The UV exposure was not strong enough to excite the dual-polarization mode, hence, the dual-wavelength laser emitted in the single polarization.

DFB-FL2 had two competing polarization modes with a relatively narrow wavelength gap of $9 \mathrm{pm}$. Further test showed that if the pump power increased from $20 \mathrm{~mW}$ to $260 \mathrm{~mW}$ gradually, the degree of the polarization (DOP) of the output increased simultaneously from 0.6 to 1 which denoted only one polarization mode and the longer wavelength laser existed. In our experiments, we found that the wavelength gap was dependent on the UV exposure, i.e. the coupling strength. The gap increased with the coupling strength. And we had obtained several DFB-FLs with different wavelength differences from $5 \mathrm{pm}$ to $15 \mathrm{pm}$. Hence for the DFB-FL fabricated with S-polarized UV laser, there was a coupling strength range to limit the UV-induced birefringence and get the stable single polarization laser output. If the coupling strength exceeded the range threshold, the two polarization modes were excited which had been analyzed extensively by Ronnekleiv [14].

For DFB-FL4, the intrinsic birefringence combining with the UV-induced birefringence was very small. Hence, the wavelength difference was very small which was usually less than $5 \mathrm{pm}$. The grating in two polarizations had almost the same coupling strength, and hence, the laser in the two polarizations had the same laser threshold and characteristics. The DOP of the DFB-FL4 output 
was less than 0.1 and almost kept constant when changing the applied tension and the pump power. More experiments on the P-UV fabricated DFB-FLs verified the stable dual polarization laser modes with very close wavelengths and powers.

DFB-FL3 was a special example. The coupling strength KL was not strong enough to produce dual polarization laser for the short cavity length of $30 \mathrm{~mm}$. The wavelength gap was about $76 \mathrm{pm}$, and it kept constant when changing the tension applied to it and the pump power. The constant DOP equal to 1 indicated the single polarization operation for the two-wavelength laser. This wavelength gap was approximately equal to the longitudinal mode gap calculated by the Fabry-Perot cavity theory. We took it as the higher mode laser excitation in DFB-FL3 because of the grating errors and phase shift errors produced in the fabrication process.

\section{Conclusions}

Four distributed feedback fiber lasers with dual-wavelength operation have been presented and investigated. The different structures and different fabrication parameters result in their different laser characteristics. The DFB-FL with two $\pi$ phase shifts may lead to a stable dual-wavelength single polarization laser. The DFB-FL with the single $\pi$ phase shift fabricated with the horizontal polarization UV laser has the stable dual-wavelength laser with two orthogonal polarization modes. The high coupling strength induced by excessive UV exposure leads to the unstable dual-wavelength laser for the DFB-FLs with a single $\pi$ phase shift fabricated with the horizontal polarization UV laser. Grating uniformity and phase-shift accuracy also have great influence on the DFB-FL characterization.

\section{Acknowledgment}

This work is financially supported by the International Science and Technology Cooperation
Program of China (2012DFA10730) and Natural Science Foundation of China (60977058).

Open Access This article is distributed under the terms of the Creative Commons Attribution License which permits any use, distribution, and reproduction in any medium, provided the original author(s) and source are credited.

\section{References}

[1] M. Ibsen, E. Ronnekleiv, G. J. Cowle, M. N. Zervas, and R. I. Laming, "Multiple wavelength all-fibre DFB lasers," Electronics Letters, 2000, 36(2): 143-144.

[2] S. Pradhan, G. E. Town, and K. J. Grant, "Dual-wavelength DBR fiber laser," IEEE Photonics Technology Letters, 2006, 18(16): 1741-1743.

[3] Y. Dai, X. Chen, J. Sun, Y. Yao, and S. Xie, "Dual-wavelength DFB fiber laser based on a chirped structure and equivalent phase shift method", IEEE Photonics Technology Letters, 2006, 18(18): 1964-1966.

[4] L. Li, A. Schulzgen, X. Zhu, J. V. Moloney, J. Albert, and N. Peyghambarian, "1 W tunable dual-wavlength emission from cascaded distributed feedback fiber lasers," Applied Physics Letters, 2008, 92(5): 051111-1-051111-3.

[5] X. Liu, "A novel dual-wavelength DFB fiber laser based on symmetrical FBG structure," IEEE Photonics Technology Letters, 2007, 19(9): 632-634.

[6] G. E. Villanueva, P. P. Millan, J. Palaci, J. L.Cruz, M. V.Andres, and J. Marti, "Dual-wavelength DFB Erbium-doped fiber laser with tunable wavelength spacing," IEEE Photonics Technology Letters, 2010, 22(4): 254-256.

[7] Z. Yin, X. Tu, S. Liu, L. Zhang, Y. Lu, and X. Chen, "Two-wavelength fiber laser based on two equivalent phase shifts," in Proc. SPIE, 2008, vol. 7278, pp. $72780 \mathrm{~J}$.

[8] L. Jia, Z. Yin, L. Zhang, and X. Chen, "Design of multiwavelength DFB fiber laser with equivalent phase shifts," Microwave and Optical Technology Letters, 2011, 53(6): 1453-1456.

[9] J. Sun, Y. Dai, X. Chen, Y. Zhang, and S. Xie, "Stable dual-wavelength DFB fiber laser with separate resonant cavities and its application in tunable microwave generation," IEEE Photonics Technology Letters, 2006, 18(24): 2587-2589.

[10] J. T. Kringlebotn, J. L. Archambault, L. Reekie, and D. N. Payne, " $\mathrm{Er}^{3+}: \mathrm{Yb}^{3+}$-codoped fiber 
distributed-feedback laser," Optics Letters, 1994, 19(24): 2101-2103.

[11] T. Erdogan and V. Mizrahi, "Characterization of UV-induced birefringence in photosensitive Ge-doped silica optical fibers," Journal of the Optical Society of America B, 1994, 11(10): 2100-2105.

[12] M. Ibsen, E. Ronnekieiv, and G. J. Cowle, "Robust high power $(>20 \mathrm{~mW})$ all-fibre DFB lasers with unidirectional and truly single polarisation outputs," in CLEO'99. Summaries of Papers Presented at the Conference on Lasers and
Electro-Optics, IEEE, USA, May, pp. 245-246, 1999.

[13] H. F. Qi, Z. Q. Song, J. Guo, J. Chang, C. Wang, and G. D. Peng, "Dual-wavelength distributed feedback fiber laser with dual symmetrical $\pi$ phase shifts," in Proc. SPIE, vol. 8924, pp. 89241G-1-89241G-4, 2013.

[14] E. Ronnekleiv, M. N. Zervas, and J. Kringlebotn, "Corrections to "modeling of polarization mode competition in fiber DFB lasers'," IEEE Journal of Quantum Electronics, 1999, 35(7): 1097-1100. 